\title{
Application of Statistical Process Control for Setting Action Thresholds as Quality Assurance of Dose Verifications in External Beam Radiotherapy
}

\author{
Philip Kioko Ndonye1, Samuel Nii Adu Tagoe ${ }^{2}$ \\ ${ }^{1}$ Oncology \& Cancer Treatment Centre, The Nairobi Hospital, Nairobi, Kenya \\ ${ }^{2}$ School of Biomedical \& Allied Health Sciences, University of Ghana/Korle Bu Teaching Hospital, Accra, Ghana \\ Email: pkndonye@yahoo.com, s.tagoe@kbth.gov.gh
}

\begin{abstract}
How to cite this paper: Ndonye, P.K. and Tagoe, S.N.A. (2022) Application of Statistical Process Control for Setting Action Thresholds as Quality Assurance of Dose Verifications in External Beam Radiotherapy. International Journal of Medical Physics, Clinical Engineering and Radiation Oncology, 11, 2235.

https://doi.org/10.4236/ijmpcero.2022.111003
\end{abstract}

Received: November 8, 2021

Accepted: January 24, 2022

Published: January 27, 2022

Copyright $\odot 2022$ by author(s) and Scientific Research Publishing Inc. This work is licensed under the Creative Commons Attribution-NonCommercial International License (CC BY-NC 4.0). http://creativecommons.org/licenses/by-nc/4.0/ (c) (i) (8) Open Access

\begin{abstract}
Purpose: To test the concept of Statistical Process Control (SPC) as a Quality Assurance $(\mathrm{QA})$ procedure for dose verifications in external beam radiation therapy in conventional and 3D Conformal Radiotherapy (3D-CRT) treatment of cervical cancer. Materials and Methods: A study of QA verification of target doses of 198 cervical cancer patients undergoing External Beam Radiotherapy (EBRT) treatments at two different cancer treatment centers in Kenya was conducted. The target doses were determined from measured entrance doses by the diode in vivo dosimetry. Process Behavior Charts (PBC) developed by SPC were applied for setting Action Thresholds (AT) on the target doses. The AT set was then proposed as QA limits for acceptance or rejection of verified target doses overtime of the EBRT process. Result and Discussion: Target doses for the 198 patients were calculated and SPC applied to test whether the action limits set by the Process Behavior Charts could be applied as QA for verified doses in EBRT. Results for the two sub-groups of $n=3$ and $n=4$ that were tested produced action thresholds which are within clinical dose specifications for both conventional AP/PA and 3D-CRT EBRT treatment techniques for cervical cancer. Conclusion: Action thresholds set by SPC were within the clinical dose specification of $\pm 5 \%$ uncertainty for both conventional AP/PA and 3D-CRT EBRT treatment techniques for cervical cancer. So the concept of SPC could be applied in setting QA action limits for dose verifications in EBRT.
\end{abstract}

\section{Keywords}

Quality Assurance, Statistical Process Control, Action Thresholds, Dose 
Verification, External Beam Radiation Therapy

\section{Introduction}

We had only one public cancer treatment center (Kenyatta National Hospital$\mathrm{KNH}$ ) in Kenya, which had been operational since 1970s. Recently (2009-2021), six private and three public hospitals, have opened modern radiotherapy facilities for cancer care and treatment. The major workload though, lies at the public hospital where the charges for cancer care and treatment are affordable by the public compared to the private hospitals. Other four public cancer centers have been proposed and are at an advanced stage to open their radiotherapy services as well.

Although cancer can be treated by surgery or chemotherapy, radiotherapy is an essential part of cancer treatment. It can be divided into EBRT and brachytherapy. Radiotherapy is needed for the treatment of $80 \%$ of all cancer patients worldwide [1] [2]. The treatment of choice depends on the type of cancer, the stage and extent of the disease among other factors. Some of the cancer treatments are given by either one treatment modality (for example, radiotherapy, surgery or chemotherapy) or by a combination of the different modalities, also depending on the type and extent of the disease.

Cervical cancer is the most commonly treated cancer by radiotherapy in Kenya as reflected on the cancer treatment statistics of 2007-2012 from the public center [3]. We proposed the QA study on cervical cancer patients due to the high frequency of the patient numbers compared to the other cancer types treated at the center.

QA is an essential part of a radiotherapy process and high accuracy is necessary to produce the desired result of tumor control rates. Also, radiation can be harmful if not used properly to protect health and minimize danger to life and property. Thus, a system of high quality and safety culture has to be developed within the course of dose delivery.

"QA in radiotherapy is concerned with all aspects of the radiotherapy process ranging from registration, diagnosis, simulation, treatment planning, dose delivery to dose verification. It is also concerned with the identification and reduction of sources of uncertainties and errors, taking into consideration the economic, medical and legal implications; as well as consideration of the need for accuracy in the radiotherapy process and avoidance of treatment errors. Also, note that random and systematic 'errors' should be called 'uncertainties'” [4].

In Kenya, to date, there are only three operational public radiotherapy centers against a population of about 48 million people. The quality of dose delivery thus may be compromised by other factors. So, there was a need now to establish a routine procedure for dose verification in radiotherapy and in vivo dosimetry is one of the methods that can be used for verification of the radiation dose deliv- 
ered to a patient in EBRT.

In Vivo dosimetry has proved to be a useful tool for QA in radiotherapy. It is the only way to check that the delivered dose corresponds to the prescribed dose [5] [6].

In Vivo dosimeters can be used to identify major deviations in the delivery of treatment and to verify and document the dose to critical structures. They can also have large uncertainties which should be assessed before using them.

In Vivo dose measurements can be divided into entrance dose measurements, exit dose measurements and intra-cavitary dose measurements.

The entrance dose for in vivo dose measurements is defined as the dose at the depth of dose maximum. It can verify the patient set-up, the radiation output, and the performance of the radiation equipment. Likewise, exit dose measurements verify the dose calculation algorithm and determine the effect of various factors like the contour of a portal treatment, patient's thickness and tissue in-homogeneities as well as calculation of absorbed dose for radiation therapy of cancer patient [7] [8].

Entrance and exit doses can be derived from diode readings by multiplication with an absorbed dose to water calibration coefficient and a number of correction factors that depend on the specific irradiation parameters used. It is also necessary to calibrate the diodes frequently due to the decrease in sensitivity with integrated dose, for example once in a few weeks, depending on the diode workload.

Uncertainties in measurements are expressed as relative standard uncertainties and the evaluation of standard uncertainties is classified into type A and type B. The method of evaluation of type A standard uncertainty is by statistical analysis of a series of observations, whereas the method of evaluation of type B standard uncertainty is based on means other than statistical analysis of a series of observations [9].

TLDs and semiconductor detectors (silicon diodes) are the types of dosimeters most commonly employed for in vivo dosimetry purposes [10]. Other systems have also been used, including film, gel dosimeters, ionization chambers and electronic devices.

Diodes have an advantage of having high sensitivity, give instant read-out and require only simple instrumentation. Their disadvantages include the exhibition of directional dependence related to the construction of the diode and its build-up cup; and for entrance and exit dose measurements, separate calibrations are required, with the diodes irradiated on both orientations.

The purpose of a QA procedure in radiotherapy is to evaluate a particular treatment delivery process. To develop a QA procedure, specifications must be defined for whether a particular instance of the QA result is acceptable or unacceptable. Radiotherapy specifications are set by requirements on the dose delivered to the patient to treat the disease [11] [12]. Within the QA process, there are random and systematic errors which can be differentiated by action thresholds developed by statistical process control as proposed by Pawlicki [13].

One goal of an optimal QA procedure is to minimize the number and magni- 
tude of systematic errors. This can be achieved quantitatively by setting action thresholds. A conventional approach for setting action thresholds is to use the mean and standard deviation of a data set obtained by the QA process [14]. Thresholds on treatment accuracy were set on the allowed difference between the treatment plan dose and the measured diode dose; and action thresholds based on the in vivo

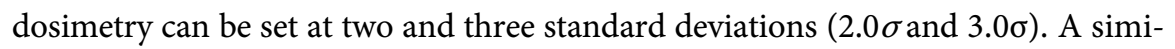
lar approach was used by Van Esch et al. [15] where 202 cases of breast cancer treatment were used to collate treatment parameters such as length, vertical table position, total dose, etc. The mean and standard deviation of each parameter are determined and action thresholds are set at about two to three times the standard deviation. If any future value was outside the threshold, then further investigation should be done.

Process behavior charts were first developed by Shewhart [16] and are the foundation of SPC. QA can be described from a process behavior viewpoint in regard to the development and characteristics of process behavior charts and their function in setting action thresholds. Process behavior charts in application to cervical cancer treatments in radiotherapy by EBRT will be given. The steps to create these charts are described as well as specifics of how to use them.

\section{Materials and Methods}

Previously, the main equipment for cervical cancer treatment by EBRT in Kenya was two Co-60 machines available at the public referral hospital (KNH). One of the two Co-60 machines, referred to as Equinox (MDS Nordion, Ottawa, Canada), was used mostly for this work. The QA equipment used for EBRT dose calibration were an Ionization chamber (NE 2581, Farmer type) \& electrometer, water phantom $(30 \times 30 \times 30 \mathrm{~cm})$, thermometer and barometer.

One diode and electrometer were used for the patients' in vivo dose verification. The diode was a cylindrical type, designed for use with photon beam energies between Cobalt-60 and $4 \mathrm{Mv}$ and manufactured by Nuclear Associates (serial No. 11623, code No. NA\#30-490).

The electrometer was manufactured by Radiation Measurements Inc. (RMI), Model No. 250 and Serial No. 250-1013D.

Patients who had qualified for radiotherapy treatment by EBRT and had accepted and consented for dose verification measurement were considered for the study. Only two measurements were done on each patient during her first, second or third day of the daily dose treatment. The two doses are the entrance dose measurement which was taken while the patient is received her AP dose and the other, the exit dose, during the opposed PA treatment with the diode fixed on the AP field for both the treatments.

The dose to be verified first, is the calculated dose at the entrance (reference dose). This dose is calculated from the patient's AP/PA separation (patient thickness) in centimeters and from the knowledge of PDDs for different field sizes. There are worldwide acceptable data of PDDs for different field sizes but we used 
the British Journal of Radiology chart of PDDs for Co-60 gamma radiation at 80 cm SSD [17].

The dose at the entrance for each field is prescribed, depending on the patient's thickness, so as to give $100 \mathrm{cGy}$ as the tumor at the mid-separation. The cervical cancer treatment volume was estimated to be approximately and within the midseparation of the patient and along the pelvic region of the body. All the patients were treated by Co-60 EBRT at KNH using two parallel opposed AP/PA fields technique.

We investigated midline target doses of cervical cancer patients with the information of patient's AP/PA separation/thickness and PDDs. The target doses are determined from entrance doses that have been measured on the central beam axis by use of a diode.

The following are some clinical considerations for patient in vivo dosimetry with diodes:

- Diode should be securely in contact with the patient skin;

- The central axis is a reproducible location;

- The placement point should be documented and reproducible for follow-up measurements.

It was noted that errors in positioning the diode for the exit dose on a patient may occur since the central axis is not visible on the exit side. These errors were minimized by placing the diode at a documented position that is reproducible on all the patients. The position of the diode for each patient would be along the patient's posterior longitudinal mid-line, and on the centre of the posterior treatment field.

The Nairobi Hospital (TNH) was the second cancer center where 90 cervical cancer patients were verified. The equipment for cervical cancer treatment by EBRT was two Varian Linear Accelerators (2300D series) that provide $6 \mathrm{MV}$ and $15 \mathrm{MV}$ photon beams. Diode in vivo dosimeters (IBA Dosimetry GmbH; Schwarzenbruck, Germany) for these photon energies were also available and were used for verification of the patient doses. Here, 3D CRT treatment technique was used for EBRT treatment of anterior and two lateral fields for each daily treatment. Only the anterior field dose was verified here for each patient and the IVD verification doses then normalized to $100 \mathrm{cGy}$ and plotted in Figure 2.

Results of the verification data from the two institutions ( $\mathrm{KNH}$ and $\mathrm{TNH}$ ) were then plotted in Figure 1 and Figure 2 and clearly indicated different positions of the four cadres.

A normal curve is a distribution of frequencies of the characteristics of interest. In the case of normal distribution, the random variable is the characteristic of interest which is usually plotted on the horizontal axis and the frequency of the characteristic plotted on the vertical axis. The normal distribution curve depends only on two parameters: the population mean, $\mu$, and the population standard deviation, $\sigma$. The shape of the normal curve is determined by the value of the population standard deviation. Normal distributions with small standard deviations 


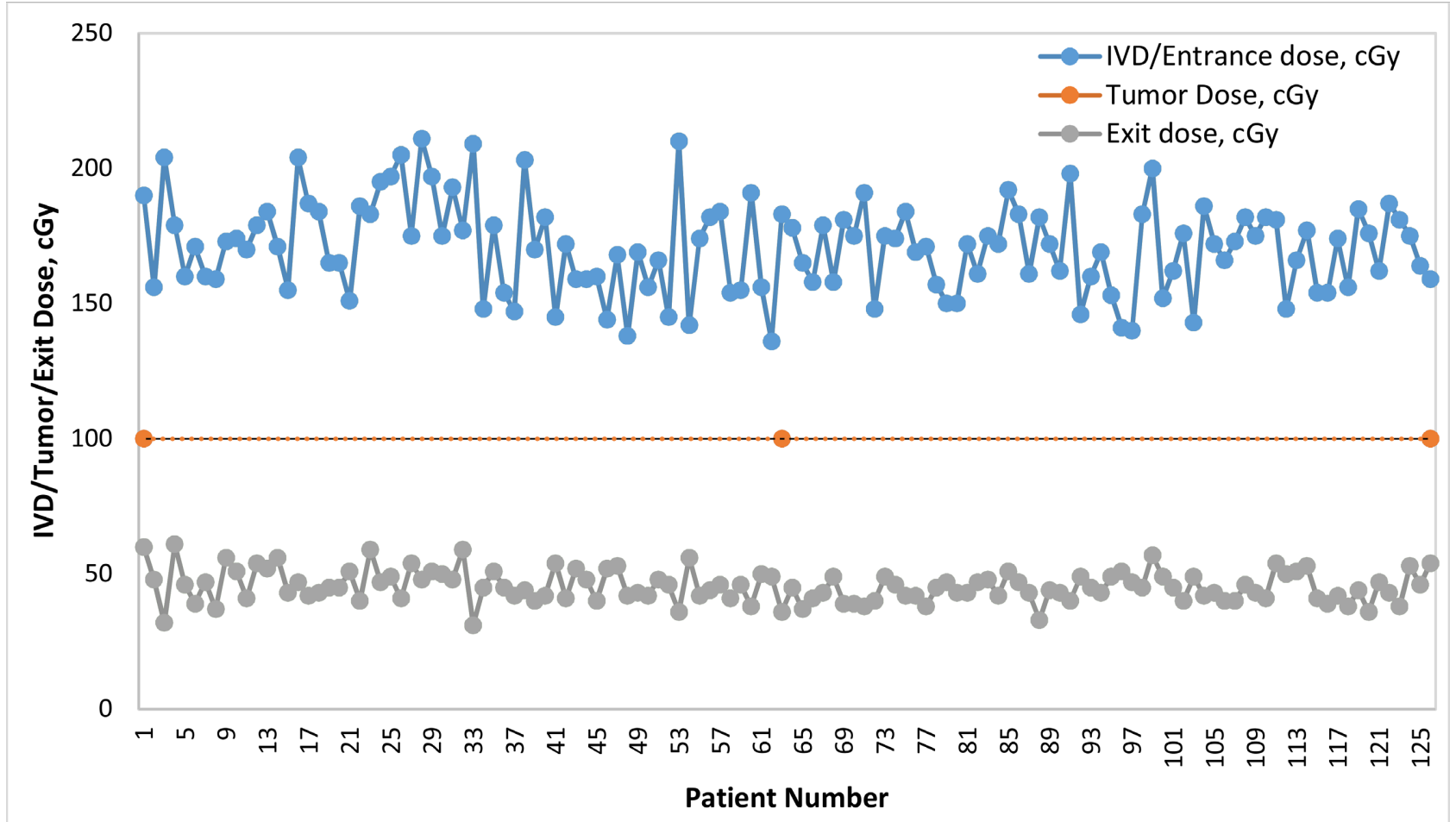

Figure 1. Graph of entrance, tumor and exit doses of 126 cervical cancer patients treated by Co-60 at KNH in 2013.

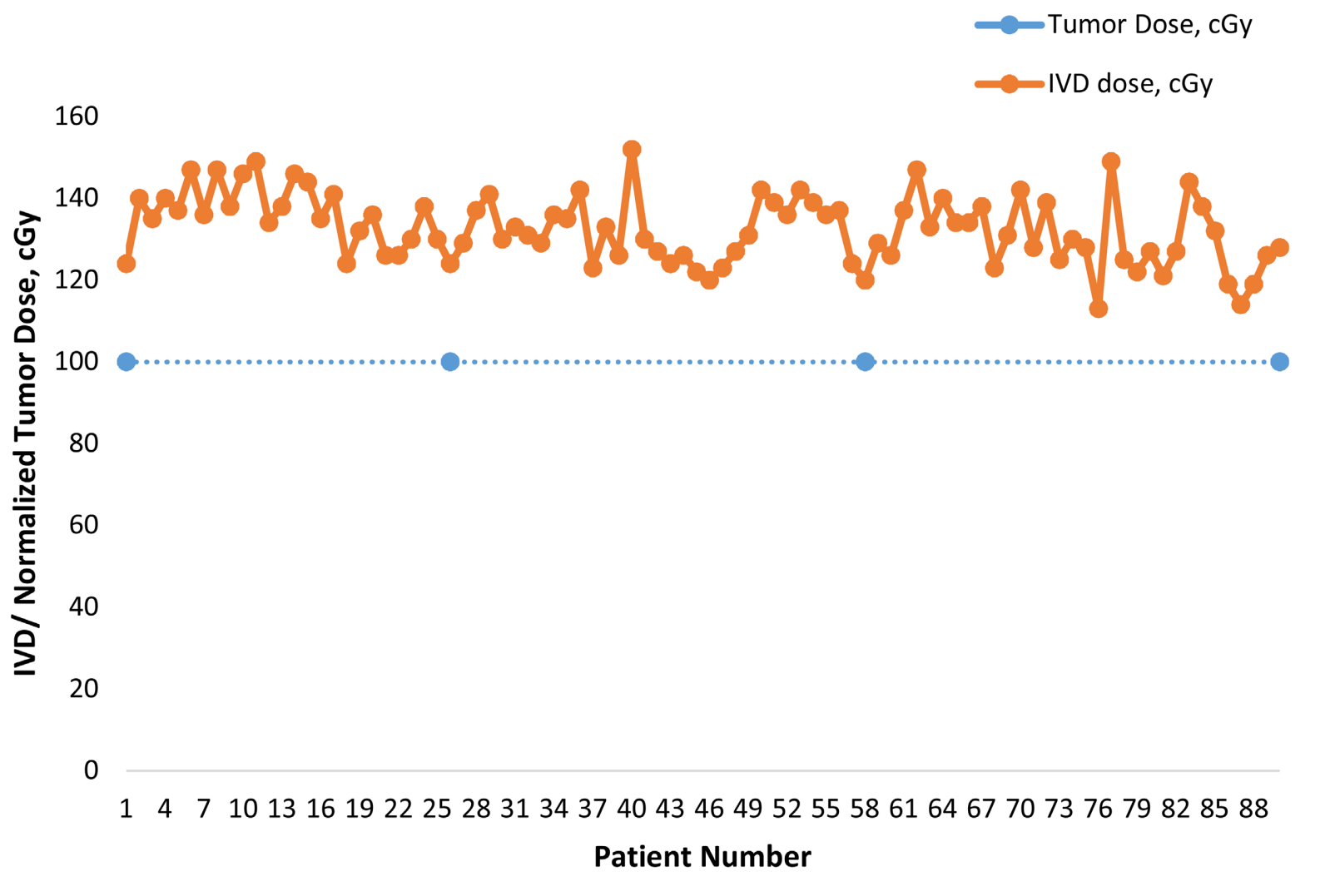

Figure 2. Graph of normalized IVD entrance and tumor dose for 90 cervical cancer patients treated at TNH in 2016. 
have narrow steep sided and peaked bell shapes. Those with large standard deviations have curves with less pronounced peaks and less steep sides. So there is a very large group of curves of varied peaks belonging to the normal curve family. Each curve differs from all the others by its mean and standard deviation. The shape of the plotted graph is an indication that the sampled population was normally distributed (Figure 3).

For the scope of this work, we will define the mean and standard deviation.

Consider the following sampled population of a normal distribution; $x_{1}, x_{2}, x_{3}, \cdots, x_{N}$, where $N=108$.

$$
\begin{aligned}
& \text { Mean, } \mu=\bar{x}=\left[x_{1}+x_{2}+x_{3}+\cdots+x_{N}\right] / N \\
& \text { Standard deviation, } \sigma=\sum_{i=1}^{N} \sqrt{[x-\bar{x}]^{2} / N}
\end{aligned}
$$

Given a QA procedure from which sequential data may be obtained, the stream of data may be used to draw inductive conclusions about the underlying process [14]. From the measurement set, the physicist may need to know when an anomalous data point is within the limits of random variation in the process or when it is due to a systematic change in the process.

An average chart can be defined to achieve this. It provides a measure of location dispersion for the data. Each chart will have an upper (UL) and a lower limit (LL) which are determined statistically and will serve as action thresholds.

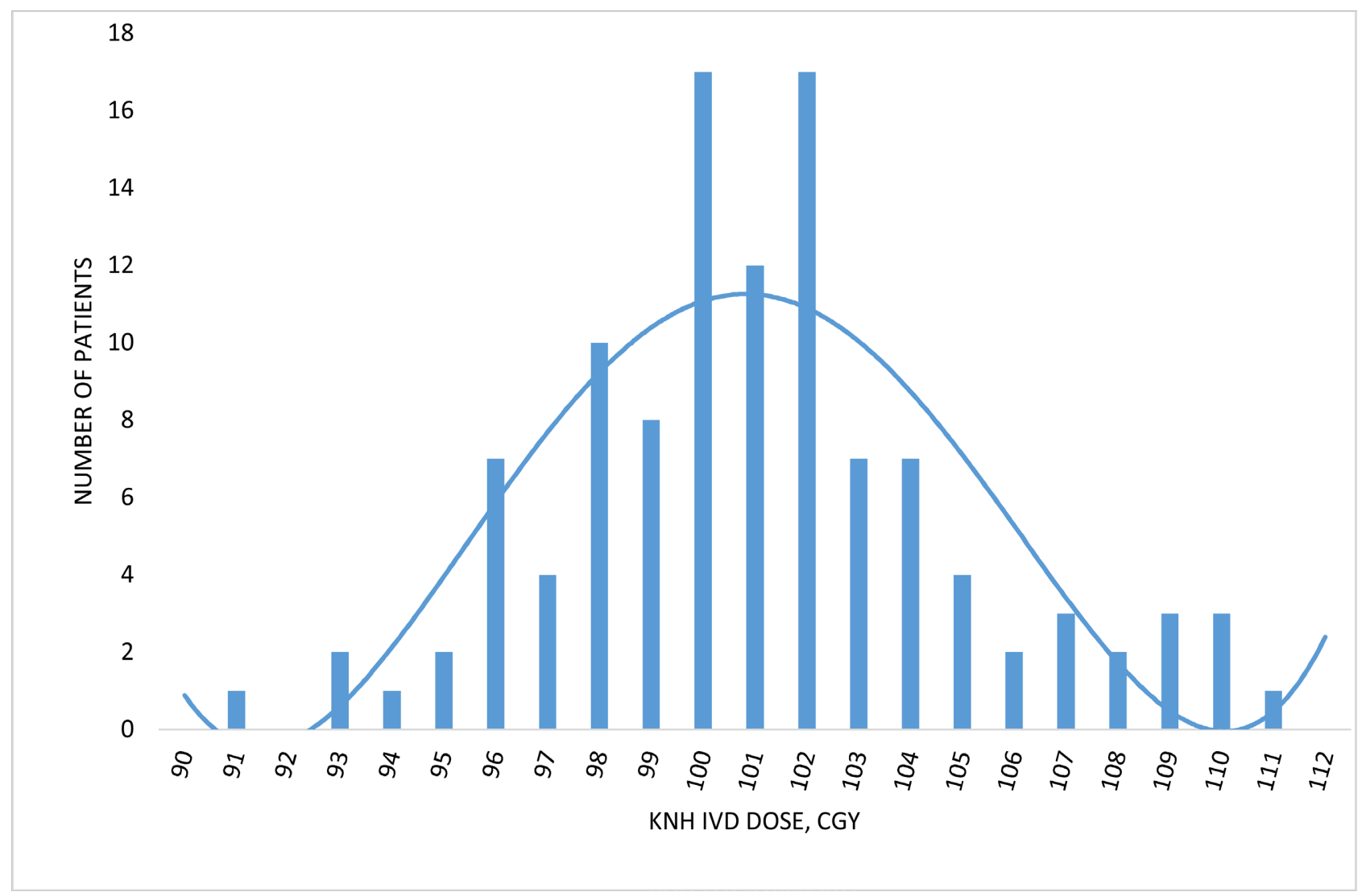

Figure 3. Histogram presentation of the target dose of the $126 \mathrm{KNH}$ cervical cancer patients sampled against their frequency. 
The procedure for creation of average chart is described in Appendix A.

Average charts have been created for the 108 patients treated and verified, Figure 4 and Figure 5.

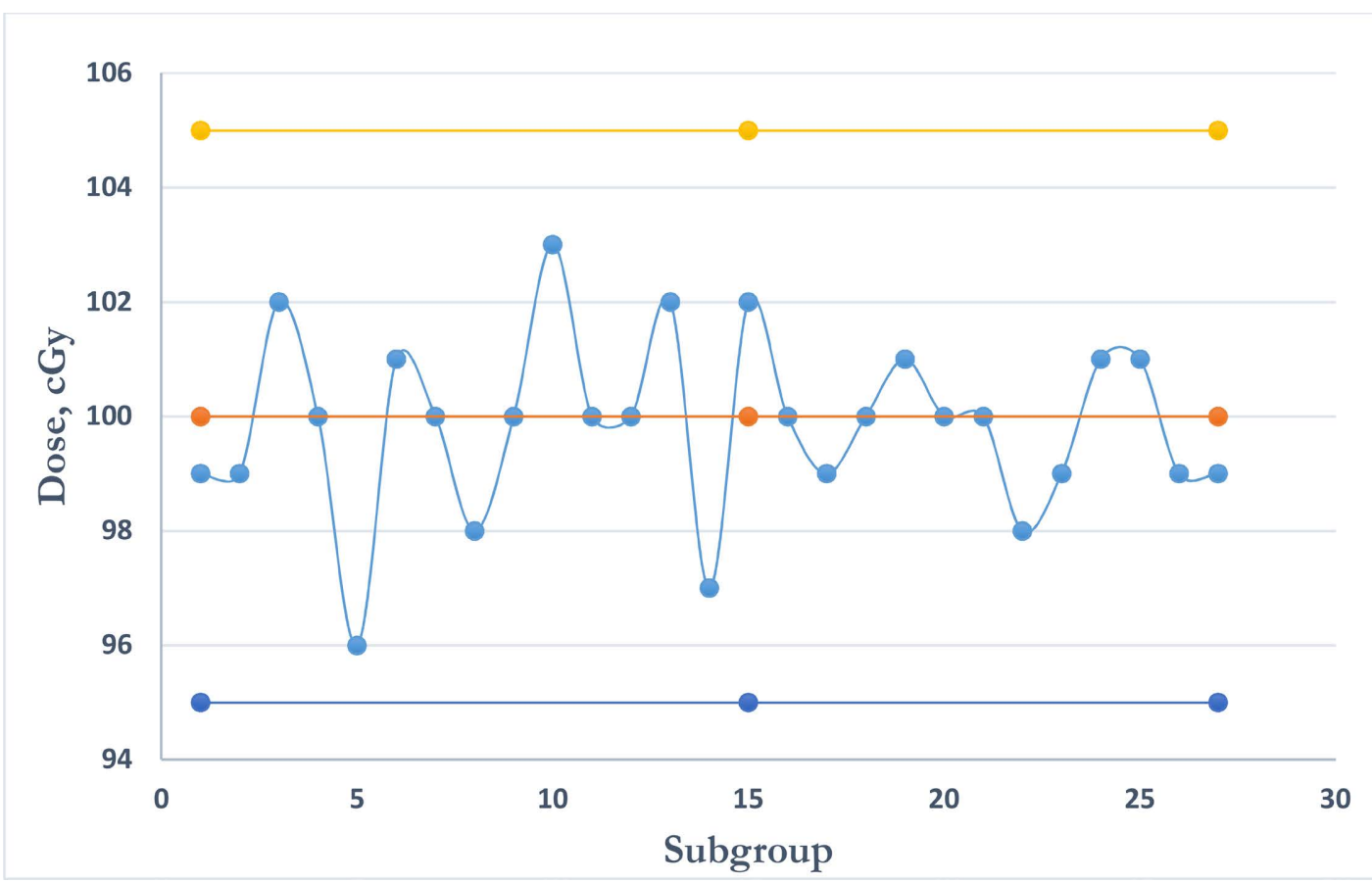

Figure 4. Average chart for KNH tumor dose data of subgroup size $n=4$. The data are sampled from the normal distribution and have mean tumor dose, $\mu=100 \mathrm{cGy}$, and standard deviation $\pm 5 \%$ (at $95 \%$ confidence interval, $2 \sigma$ ) as determined by SPC.

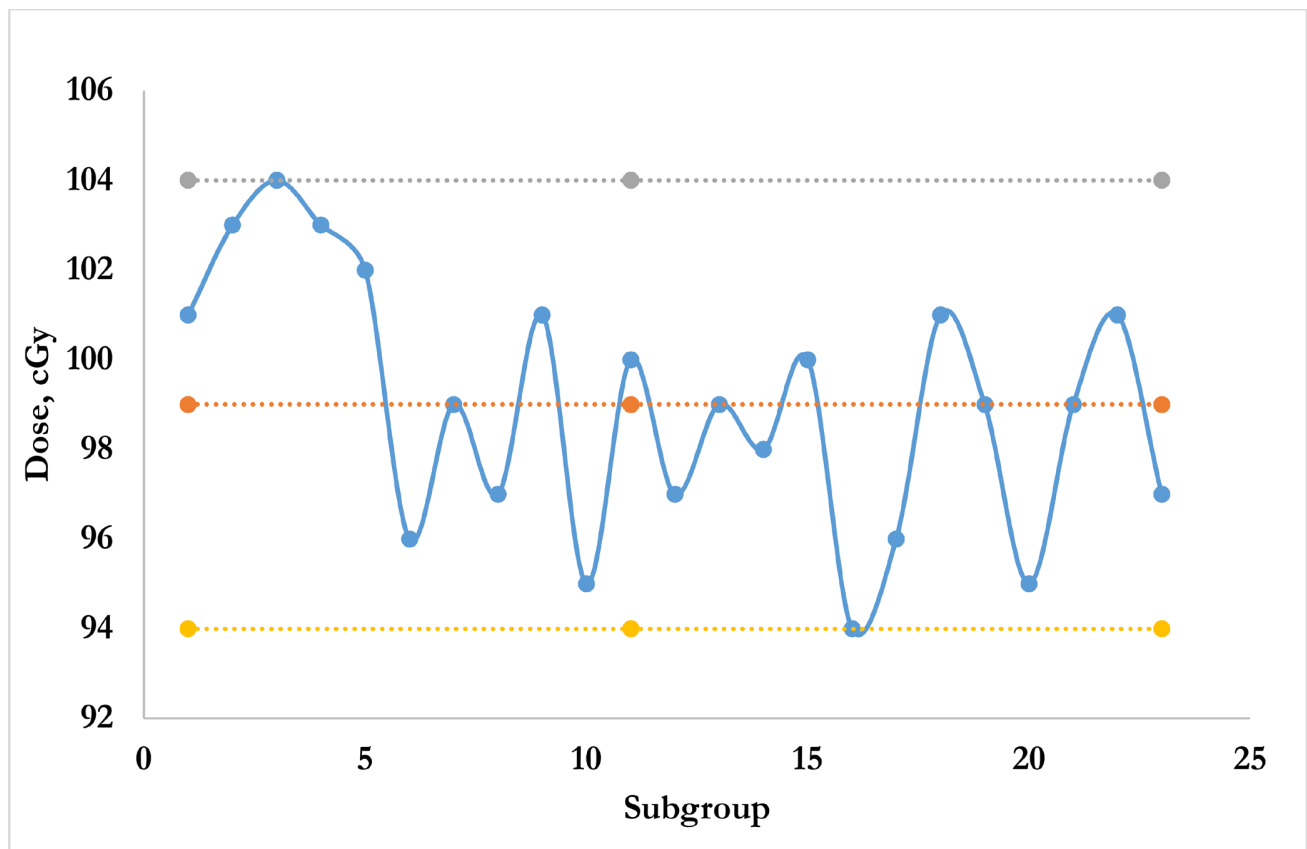

Figure 5. Average chart for TNH tumor dose data of subgroup size $n=4$. The data are sampled from the normal distribution and have mean tumor dose, $\mu=99 \mathrm{cGy}$, and standard deviation $\pm 5 \%$ (at 95\% confidence interval, $2 \sigma$ ). 


\section{Results and Discussion}

Patient data of entrance and exit dose were determined by use of a diode in vivo dosimetry system after the required calibrations. At $\mathrm{KNH}$, measurements of patients' AP/PA separation were also taken for purposes of calculating the reference dose. Target doses for the 108 patients were then calculated from their respective entrance doses. Histogram graph of the target dose against frequency is presented in Figure 3, and is bell-shaped with prolonged extensions on either side. This shape is an indication of data from a normal distribution with a mean dose of 100 cGy and standard Deviation of 3.3. This data was then used to compute process behavior charts, since it qualifies to be from a normal distribution. Following Appendix A guidelines, one is then able to determine the action thresholds from both process behavior charts and standard uncertainty for subgroup size, $n=4$. Points up to subgroup No.15 (60 samples) on subgroup size $n=4$, were used for determination of action thresholds. These results are presented in Figure 4, and Figure 5 for subgroup size $n=3$.

After determination of the action thresholds, it was clear that the system dose delivery for the patients was stable, since no point was outside the action limits. If a point falls outside the action limits in future after the action limits have been set, then action ought to be taken in order to rectify the situation. Then after rectification, a repeat verification measurement has to be done and should be within the action limits in order to be acceptable. So the action limits set by the SPC would act as thresholds for a measured target dose and would be acceptable if it does not fall outside the set limits.

\section{Conclusions}

Process behavior charts determined by statistical process control of a radiotherapy quality assurance process have been used to estimate the uncertainty of dose delivery to the target volume of cancer patients from two cancer centers $(\mathrm{KNH}$ and $\mathrm{TNH}$ ) in Kenya.

The results determined at $95 \%$ confidence interval $(2 \sigma)$ show a mean target dose of 100 cGy per field with uncertainty limits set by SPC at $\pm 5 \%$ and $4 \%$ for the limits determined by standard uncertainty, for the subgroup size $n=4$. Similarly, for the same data and with the subgroup size of $n=3$, the results are also acceptable with a mean target dose of $100 \mathrm{cGy}$ per field and with a standard deviation (at $95 \%$ confidence interval, $2 \sigma$ ) of $\pm 6 \%$ and $\pm 4 \%$ as determined by SPC and by standard uncertainty respectively. The limits set here are within the clinical requirement of $\pm 5 \%$ uncertainty for conventional AP/PA and 3D-CRT treatment techniques for cervical cancer. Thus, SPC can be applied as a QA procedure for acceptance/rejection of a measured target dose of an external beam radiotherapy treatment.

\section{Acknowledgements}

I acknowledge my Institutions' Ethics and Research Committee (Kenyatta Na- 
tional Hospital, University of Nairobi, and The Nairobi Hospital) for accepting and allowing me to do my $\mathrm{PhD}$ research project at the instructions.

\section{Conflicts of Interest}

The authors declare no conflicts of interest regarding the publication of this paper.

\section{References}

[1] Gadhi, M.A., Fatmi, S., Chughtai, G.M., et al. (2016) Verification of Absorbed Dose Using Diodes in Co-60 Radiation Therapy. Australasian Physical \& Engineering Sciences in Medicine, 39, 211-219.

[2] Janaki, M.G., Kadam, A.R., Mukesh, S., et al. (2010) Magnitude of Fatique in Cancer Patients Receiving Radiotherapy and Its Short Term Effect on Quality of Life. Journal of Cancer Research and Therapeutics, 6, 22-26.

[3] Ndonye, P.K. (2018) Advances in Treatment of Cancer by Brachytherapy in Kenya, in Particular, Prostate Cancer. Journal of Medical Physics and Applied Sciences, 3, 1-8.

[4] Followill, D., Van Dyk, J. and Palta, J.R. (2007) Quality Assurance: ANL Talk, Quality Assurance Principles. Argonne National Laboratories, Chicago.

[5] Essers, M. and Mijneer, B.J. (1999) In Vivo Dosimetry during External Beam Radiotherapy. International Journal of Radiation Oncology, Biology, Physics, 43, 245-259. https://doi.org/10.1016/S0360-3016(98)00341-1

[6] Florino, C., Corletto, D., Mangili, P., et al. (2000) Quality Assurance by Systematic In Vivo Dosimetry; Results on a Large Cohort of Patients. Radiotherapy \& Oncology, 56, 85-95. https://doi.org/10.1016/S0167-8140(00)00195-X

[7] AAPM (2005) Diode In Vivo Dosimetry for Patients Receiving External Beam Radiation Therapy. American Association of Physicists in Medicine.

[8] ESTRO (2001) Practical Guidelines for the Implementation of in Vivo Dosimetry with Diodes in External Beam Radiotherapy with Photon Beams (Entrance Dose). Physics for Clinical Radiotherapy. 1st Edition, European Society for Therapeutic Radiology and Oncology, Brussels.

[9] Podgorsak, E.B. (2005) Radiation Oncology Physics: A Handbook for Teachers and Students. International Atomic Energy Agency, Vienna, 72-74.

[10] IAEA (2013) Development of Procedures for in Vivo Dosimetry in Radiotherapy. IAEA Human Health Report No. 8. International Atomic Energy Agency, Vienna.

[11] Boyer, A.L. and Schultheiss, T. (1988) Effects of Dosimetry and Clinical Uncertainty on Complication-Free Local Tumor Control. Radiotherapy \& Oncology, 11, 65-71. https://doi.org/10.1016/0167-8140(88)90046-1

[12] Brahme, A. (1984) Dosimetric Precision Requirements in Radiation Therapy. Acta Radiologica: Oncology, 23, 379-391. https://doi.org/10.3109/02841868409136037

[13] Pawlicki, T., Whitaker, M. and Boyer, A.L. (2005) Statistical Process Control for Radiotherapy Quality Assurance. Medical Physics, 32, 2777-2786. https://doi.org/10.1118/1.2001209

[14] Cozzi, L. and Fogliata-Cozzi, A. (1998) Quality Assurance in Radiation Oncology. A Study of Feasibility and Impact on Action Levels of an in Vivo Dosimetry Program during Breast Cancer Irradiation. Radiotherapy \& Oncology, 47, 29-36. https://doi.org/10.1016/S0167-8140(97)00202-8

[15] Van Esch, A., Bogaerts, R., Kutcher, G.J. and Huyskens, D. (2000) Quality Assurance 
by Identifying Standards and Monitoring Treatment Preparation. Radiotherapy \& Oncology, 56, 109-115. https://doi.org/10.1016/S0167-8140(00)00190-0

[16] Shewhart, W.A. (1931) Economic Control of Quality of Manufactured Product. D. Van Nostrand Company, Inc., New York.

[17] British Institute of Radiology (1996) Central Axis Depth Dose Data for Use in Radiotherapy. The British Journal of Radiology, 25. 


\section{Appendix A. Theory/Calculation}

\section{SPC Application to QA in Radiotherapy}

Given a QA procedure from which sequential data may be obtained, the stream of data may be used to draw inductive conclusions about the underlying process. From the measurement set, the physicist may need to know when an anomalous data point is within the limits of random variation in the process or when it is due to a systematic change in the process.

Two charts can be defined to achieve this; one that provides a measure of location for the data and one that provides a measure of dispersion for the data. The average and range charts are used here for the location and dispersion of the data respectively; although other location and dispersion statistics could be used with equal effectiveness. Each chart will have an upper and a lower limit which are determined statistically and will serve as action thresholds.

Several steps are available for creation of the charts:

1) Obtain data from a $Q A$ process;

2) Compute summary statistics of the data;

3) Determine process parameters by obtaining limits;

4) Use process parameters to characterize process outcomes.

First, organize the data into periodic subgroups of $2,3,4, \ldots$, or 100 ; so that you can monitor the location or dispersion of the data generated by the treatment process. Several measurements are obtained under the same conditions; and these are the basis of a subgroup to be used as separate data that are subject to analysis.

If the subgroups are statistically consistent over time, then the process that created those subgroups is stable; that is, subject to only random variations. If the subgroups are not consistent over time, then the process under consideration is unstable and subject to both random and systematic variations. A process that contains both random and systematic variations is unpredictable and a process that displays only random variations is predictable within the magnitude of random errors in the process. Action thresholds set by process behavior limits will distinguish between the two sources or error. Data points within the thresholds are within the range of random errors and may not have readily assignable cause where as those data outside the thresholds will be likely to have assignable cause for the systematic error.

\section{Creation of Process Behavior Charts}

A QA procedure for dose verification of cervical cancer treatments by EBRT was used for data acquisition of 108 patients treated on Equinox Co-60 Teletherapy machine at $\mathrm{KNH}$. The technique of cervical cancer treatment here is by application of two parallel opposed AP/PA fields for a daily dose of $200 \mathrm{cGy}$. All the patients were treated here with an aim of giving a dose of $100 \mathrm{cGy}$ to the target from each field. 
Numerical data acquired; $x_{1}, x_{2}, x_{3}, \cdots, x_{N}$, where $N=108$.

Subgroups of this data were then created; $x_{1}, x_{2}, x_{3}, \cdots, x_{n}$, where $n<N ; n=4$.

The average of a subgroup is $x$ and the range value in a subgroup is $R$. The general principle when choosing a subgroup size is to ensure subgroup homogeneity, that is, ensure the same quantity is being measured in the same way for each subgroup. To monitor a process, two process behavior charts will be created; an average chart for subgroup averages, $\bar{x}$, and a range chart for subgroup ranges, $R$. The centre-line for the subgroup average chart is $\overline{\bar{x}}$, which is the average of all subgroup averages. The centre-line for the subgroup range chart is $\bar{R}$, which is the average of all the subgroup ranges. The average chart will have an upper threshold $\left(A_{u}\right)$, centre-line $\left(A_{c}\right)$, and lower threshold $\left(A_{l}\right)$ defined as:

$$
\begin{aligned}
& A_{u}=\overline{\bar{x}}+3 \frac{\bar{R}}{d_{2} \sqrt{n}} \\
& A_{c}=\overline{\bar{x}} \\
& A_{1}=\overline{\bar{x}}-3 \frac{\bar{R}}{d_{2} \sqrt{n}}
\end{aligned}
$$

where the factor 3 is added as an economical margin for the limits. Similarly the range chart will have an upper threshold $\left(R_{u}\right)$, centre-line $\left(R_{c}\right)$ and lower threshold $\left(R_{l}\right)$ defined as:

$$
\begin{aligned}
& R_{u}=\left\{1+3 \frac{d_{3}}{d_{2}}\right\} \bar{R} \\
& R_{c}=\bar{R} \\
& R_{l}=\left\{1-3 \frac{d_{3}}{d_{2}}\right\} \bar{R}
\end{aligned}
$$

The quantities $d_{2}$ and $d_{3}$ depend on the subgroup size $n$, and are available in Table A1 of Appendix B.

The action thresholds determined by process behavior charts on the average chart are series 5 and 6 , while series 3 and 4 are limits calculated by two standard uncertainties, where $s(x)$, (from Appendix A) is:

$$
s(x)=\sqrt{\left[n \sum x^{2}-\left(\sum x\right)^{2}\right] / n(n-1)}
$$




\section{Appendix B}

The following table provides a list of bias correction factors, $d_{2}$ and $d_{3}$ for different subgroup sizes $n$. The bias correction factors establish a relationship between the standard deviation for the original distribution and the range distribution [13].

Table A1. List of bias correction factors, $d_{2}$ and $d_{3}$, for different subgroup sizes, $n$.

\begin{tabular}{|c|c|c|}
\hline Subgrop size, $n$ & $d_{2}$ & $d_{3}$ \\
\hline 2 & 1.28 & 0.8525 \\
\hline 3 & 1.693 & 0.8884 \\
\hline 4 & 2.059 & 0.8798 \\
\hline 5 & 2.326 & 0.8641 \\
\hline 6 & 2.534 & 0.848 \\
\hline 7 & 2.704 & 0.8332 \\
\hline 8 & 2.847 & 0.8198 \\
\hline 9 & 2.97 & 0.8078 \\
\hline 10 & 3.078 & 0.7971 \\
\hline 11 & 3.173 & 0.7873 \\
\hline 12 & 3.258 & 0.7785 \\
\hline 13 & 3.336 & 0.7704 \\
\hline 14 & 3.407 & 0.763 \\
\hline 15 & 3.472 & 0.7562 \\
\hline 20 & 3.735 & 0.7287 \\
\hline 25 & 3.931 & 0.7084 \\
\hline 30 & 4.086 & 0.6927 \\
\hline 40 & 4.322 & 0.6692 \\
\hline 50 & 4.498 & 0.6521 \\
\hline 100 & 5.015 & 0.6052 \\
\hline
\end{tabular}

